\title{
Advanced Hormone-Sensitive Breast Cancer: Overcoming Resistance
}

Presented by Ingrid A. Mayer, MD, MSCI

\begin{abstract}
Novel strategies to overcome resistance to endocrine therapy are on the horizon, especially PI3K inhibitors, CDK 4/6 inhibitors, and agents targeting methylation. However, many, if not most, patients with progressive estrogen receptor-positive breast cancer will continue to show response to multiples lines of endocrine therapy alone. Which patient subsets will be candidates for the emerging agents is still unclear, but in the future, subtype of the tumor, mutational status, and treatment goals will be part of the equation. Always, it will be necessary to individualize treatment. (J Natl Compr Canc Netw 2015;13:655-657)
\end{abstract}

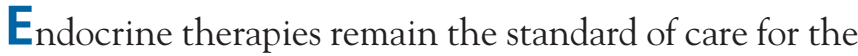
treatment of advanced hormone-sensitive breast cancer, but on the horizon are novel strategies that may help overcome the resistance these tumors acquire, according to Ingrid Mayer, MD, MSCI, who spoke on the topic at the NCCN 20th Annual Conference.

Dr. Mayer, Associate Professor of Medicine and Clinical Director of the Breast Cancer Program at Vanderbilt-Ingram Cancer Center, described promising targeted agents but emphasized, "just because we will have targeted drugs available, does not mean we will need to use them in all our patients."

These novel therapies will need to be individualized, based on the profile of resistance, treatment goals, and potentially the tumor mutational profile. Meanwhile,

Presented by Ingrid A. Mayer, MD, MSCl, Associate Professor of Medicine and Clinical Director of the Breast Cancer Program at Vanderbilt-Ingram Cancer Center, Nashville, Tennessee.

Dr. Mayer has disclosed that she has served as an advertising board consultant for Novartis and Genentech, and has received research support from Novartis.

Correspondence: Ingrid A. Mayer, MD, MSCl, Vanderbilt-Ingram Cancer Center, 2220 Pierce Avenue, 777 PRB, Nashville, TN 37232.

E-mail: ingrid.mayer@vanderbilt.edu with current treatments, many patients can continue to benefit from multiple lines of endocrine therapy, with chemotherapy generally reserved for patients with visceral involvement, she said.

\section{Degree of Endocrine Resistance Guides Treatment}

For some time, researchers have focused on targeting mechanisms of endocrine resistance, which appear to differ between tumors that are primarily (or de novo) resistant to endocrine therapy and those that develop resistance over time (acquired or secondary resistance).

"The concept of endocrine resistance helps us make decisions regarding the next line of treatment," she noted.

The definition of endocrine resistance in advanced breast cancer is evolving. Primary (de novo) resistance is defined by progressive disease within 6 months of starting treatment with endocrine therapy in the metastatic setting, or the development of metastatic recurrence during or shortly after completing adjuvant therapy. Secondary (acquired) resistance is defined by an initial response to endocrine therapy in the adjuvant and metastatic settings, with disease progression in the metastatic setting occurring 6 months or later.

Essentially, patients with primary resistance may need an additional agent from the start. Conversely, patients with acquired, secondary resistant disease may be sufficiently treated with endocrine agents at first- and even second-line therapy and only require additional agents at later disease progression (Figure 1).

\section{Genetic Profile of Resistance}

Genetic alterations are seldom seen in endocrine therapy-sensitive tumors but may be abundant in endocrine 
Mayer

\section{Patient with $\mathrm{ER}^{+} \mathrm{MBC}$ should be treated with endocrine therapy unless: \\ - Progression of disease after 3 lines of endocrine therapy \\ - Visceral crisis}

\begin{tabular}{|l|l|l|}
\hline Setting & Treatment choice & Reference \\
\hline 1st line, no prior ET in the adjuvant setting & Fulvestrant (500)+ aromatase inhibitor & SWOG-SO026 \\
\hline $\begin{array}{l}\text { 1st line, prior ET in the adjuvant setting } \\
\mathbf{3 6 - 1 2} \text { months prior to MBC }\end{array}$ & $\begin{array}{l}\text { Al or Fulvestrant (500), clinical trials with endocrine therapy } \\
\text { (PI3K inhibitors, CDK4/6 inhibitors, etc.) }\end{array}$ & FIRST \\
\hline $\begin{array}{l}\text { 1st line, prior ET in the adjuvant setting } \\
\mathbf{5 6 - 1 2} \text { months prior to MBC }\end{array}$ & $\begin{array}{l}\text { Exemestane + everolimus, tamoxifen + everolimus, } \\
\text { Fulvestrant (500), clinical trials with endocrine therapy (PI3K } \\
\text { inhibitors, CDK4/6 inhibitors, etc.) }\end{array}$ & $\begin{array}{l}\text { BOLERO-2, TAMRAD, } \\
\text { CONFIRM }\end{array}$ \\
\hline 2nd line & $\begin{array}{l}\text { Exemestane + everolimus, tamoxifen + everolimus, } \\
\text { Fulvestrant (500), clinical trials with endocrine therapy (PI3K } \\
\text { inhibitors, CDK4/6 inhibitors, etc.) }\end{array}$ & $\begin{array}{l}\text { BOLERO-2, TAMRAD, } \\
\text { CONFIRM }\end{array}$ \\
\hline 3rd line & $\begin{array}{l}\text { Exemestane + everolimus, tamoxifen + everolimus, clinical } \\
\text { trials with endocrine therapy (PI3K inhibitors, CDK4/6 } \\
\text { inhibitors, etc.) }\end{array}$ & BOLERO-2, TAMRAD \\
\hline$>3 r d$ line & $\begin{array}{l}\text { Clinical trials with endocrine therapy (PI3K inhibitors, } \\
\text { CDK4/6 inhibitors, etc.); chemotherapy }\end{array}$ & NCCN, ABC-1 \\
\hline
\end{tabular}

Figure 1 Treatment landscape for estrogen receptor-positive metastatic breast cancer.

Abbreviations: Al, aromatase inhibitor; ET, endocrine therapy, $\mathrm{MBC}$, metastatic breast cancer.

therapy-resistant tumors. In de novo resistance, loss of estrogen receptors (ER) or loss of amplification of co-receptors and co-amplifiers, as well as activation of the cyclin D pathway, are common mechanisms of resistance. For patients with acquired resistance who initially show response to endocrine therapy, $\mathrm{PI} 3 \mathrm{~K}$ pathway activation is a common resistance mechanism.

Point mutations are associated with resistance to conventional endocrine therapies, and they can be detected after exposure to endocrine therapy. Several point mutations in the ligand-binding domain of ESR1 have been identified; they confer constitutive ligand-independent activation of ER transcription and ER- $\alpha$ expression and may mediate antiestrogen resistance.

"This is important, because novel agents can tap into some of these mutations," Dr. Mayer explained.

One of these is ARN-810, a novel selective ER- $\alpha$ antagonist that induces proteasomal-mediated degradation of ER- $\alpha$. This drug is now in phase II trials, and may be found to be active in tumors with the ESR1 mutation, whereas conventional endocrine therapies are not. ${ }^{1}$

\section{PI3K Pathway Inhibitors}

Several inhibitors are currently in development for growth factor receptor and intracellular targets. In selected patients, these drugs would be adjuncts to endocrine therapy.
Mutations within the PI3K/mTOR pathway are seen in more than one-third of ER-positive tumors. In early-stage breast cancer, these mutations confer a good prognosis, but the opposite seems to be true in the metastatic setting after exposure to endocrine therapy. In this setting, the pathway activation conferred by PIK3CA mutations may behave as a mechanism of antiestrogen resistance. Therefore, PI3K pathway inhibitors may have more relevance for patients with acquired endocrine therapy resistance, Dr. Mayer indicated.

BOLERO-2 is a phase III clinical trial that showed progression-free survival benefit for the addition of the mTOR inhibitor everolimus to exemestane in patients with disease refractory to aromatase inhibitors. ${ }^{2}$ Interestingly, this benefit was seen regardless of PIK3CA mutation status. FDA approval was granted on the basis of this study, but it is worth noting that no overall survival advantage was seen. However, everolimus remains an option in secondor third-line endocrine treatment of ER-positive metastatic breast cancer.

"Agents blocking PI3K, either pan or selective PI3K inhibitors, are currently in phase III clinical trials and have so far shown an acceptable toxicity profile," Dr. Mayer noted. Other agents targeting this pathway are the TORC1/TORC2 inhibitors, dual $\mathrm{PI} 3 \mathrm{~K} / \mathrm{mTOR}$ inhibitors, and AKT inhibitors, but these agents are not so far in development due to some toxicity concerns. 
Preclinically, the pan-PI3K inhibitor buparlisib combined with fulvestrant has shown robust tumor suppressive activity. Clinically, it has also shown some preliminary activity in combination with letrozole, with an acceptable toxicity profile, in a somewhat heavily pretreated patient population. "We saw that a subset clearly had a dramatic benefit in combination with endocrine therapy, regardless of PIK3CA mutation presence" she said. Data are now awaited from the phase III BELLE-2 and BELLE-3 trials.

The pan-PI3K inhibitor pictilisib ${ }^{4}$ appears to show less benefit in a recent phase II trial, but it is worth noting that only a small proportion of patients had hyperglycemia, an on-target effect for this class of drugs. However, the alpha-specific PI3K inhibitor BYL719 and the beta-sparing PI3K inhibitor GDC0032 appear promising in patients with PIK3CA mutations. ${ }^{5}$

"The jury is still out on whether PI3K inhibitors will be useful in advanced ER-positive breast cancer," Dr. Mayer commented. At present, she said, "The combination of endocrine therapy with PI3K pathway inhibitors may further benefit ER-positive, PIK3CA-mutant breast cancers, particularly, and not exclusively, those at advanced stage."

\section{Targeting CDK 4/5-Cyclin D1-E2F and Methylation}

Cyclin-dependent kinases (CDKs) are key regulators of the cell cycle, and numerous inhibitors are currently in development. One of these, the CDK 4/6 inhibitor palbociclib, recently showed promising results in the phase II PALOMA-1/TRIO-18 trial. $^{6}$ Added to letrozole, palbociclib reduced the risk of disease progression by $51 \%(P<.0004)$, leading to an accelerated FDA approval. The phase III PALOMA-2 is seeking to confirm the benefit, while PALOMA-3 will evaluate palbociclib in second-line therapy in combination with fulvestrant.

"Palbociclib is an appropriate option to consider in combination with endocrine therapy, particularly for patients appearing to have primary endocrine resistance," Dr. Mayer suggested. It is worth noting that, because of its side effect profile (neutropenia, gastrointestinal toxicity), palbociclib may not be the best choice for all patients.

Finally, targeting methylation through inhibition of histone deacetylase could reverse resistance by opening up the DNA structure and enabling the transcription of ER. In the phase II ENCORE 301 study, entinostat plus exemestane as second-line treatment reduced deaths by $41 \%(P=.036) .{ }^{7}$ This "outside the box strategy" is being further explored and appears promising. An ECOG-ACRIN phase III trial is now ongoing, Dr. Mayer indicated.

Meanwhile, she emphasized that not all patients with ER-positive disease that progresses after firstline treatment will be candidates for these novel approaches because of drug toxicity. "We can be thankful for these drugs. They are great options, but not all patients will need them," she said. Honoring treatment goals (survival improvement, palliation of symptoms, and maintenance of quality of life) takes precedence at an individual level.

\section{References}

1. Bardia A, Dickler MN, Mayer IA, et al. Phase I study of ARN-810, a novel and potent oral selective estrogen receptor degrader, in postmenopausal women with metastatic estrogen receptor positive, HER2- breast cancer [abstract]. Presented at the 2014 San Antonio Breast Cancer Symposium; December 9-13, 2014, San Antonio, TX. Abstract P1-13-01.

2. Hortobagyi GN, Piccart-Gebhart MJ, Rugo HS, et al. Correlation of molecular alterations with efficacy of everolimus in hormone receptorpositive, HER2-negative advanced breast cancer: results from BOLERO-2 [abstract]. Presented at the ASCO Annual Meeting; May 31-June 4, 2013; Chicago, IL. Abstract LBA509.

3. Mayer IA, Abramson VG, Isakoff SJ, et al. Stand up to cancer phase Ib study of pan-phosphoinositide-3-kinase inhibitor buparlisib with letrozole in estrogen receptor-positive/human epidermal growth factor receptor 2-negative metastatic breast cancer. J Clin Oncol 2014;32:1202-1209.

4. Krop I, Johnston S, Mayer IA, et al. FERGI phase II study of PI3K inhibitor pictilisib (GDC-0941) plus fulvestrant vs fulvestrant plus placebo in patients with ER+, aromatase inhibitor-(AI)-resistant advanced or metastatic breast cancer-part 1 results [abstract]. Presented at the 2014 San Antonio Breast Cancer Symposium; December 9-13, 2014, San Antonio, TX. Abstract S2-02.

5. Mayer IA, Abramson VG, Balko JM, et al. SU2C phase Ib study of the PI3K $\alpha$ inhibitor BYL719 with letrozole in ER+/HER2- metastatic breast cancer [abstract]. Presented at the ASCO Annual Meeting; May 30-June 3, 2014; Chicago, IL. Abstract 516.

6. Finn RS, Crown JP, Lang I, et al. Results of a randomized phase 2 study of PD 0332991, a cyclin-dependent kinase (CDK) $4 / 6$ inhibitor, in combination with letrozole vs letrozole alone for first-line treatment of ER+/HER2- advanced breast cancer (BC) [abstract]. Cancer Res 2012;72(24 Suppl):Abstract nr S1-6.

7. Yardley DA, Ismail-Khan RR, Melichar B, et al. Randomized phase II, double-blind, placebo-controlled study of exemestane with or without entinostat in postmenopausal women with locally recurrent or metastatic estrogen receptor-positive breast cancer progressing on treatment with a nonsteroidal aromatase inhibitor. J Clin Oncol 2013;31:2128-2135. 